\title{
Martin boundary for homogeneous riemannian manifolds of negative curvature at the bottom of the spectrum
}

Ewa Damek, Andrzej Hulanicki and Roman Urban

\section{Introduction.}

Let $M$ be a manifold and let $\mathcal{L}$ be a subelliptic second order differential operator on $M$. Positive $\mathcal{L}$-harmonic functions have been intensively studied for many decades. In particular, if $M$ has negative curvature and $\mathcal{L}$ is coercive (i.e. there is a positive $\varepsilon$ such that $\mathcal{L}+\varepsilon I$ admits the Green function), the Martin boundary has been described by A. Ancona $[\mathrm{A}]$, and earlier by M. Anderson and Schoen $[\mathrm{AS}]$ in the case when $\mathcal{L}$ is the Laplace-Beltrami operator. If $\mathcal{L}$ is noncoercive, the situation is much more complicated, there are no results like in $[\mathrm{A}]$, so various particular cases are of interest.

In this paper we treat noncoercive operators on simply connected homogeneous manifolds of negative curvature. J. Wolf [W] and E. Heintze [Hei] proved that such a manifold is isometric with a solvable Lie group $S=N A$, being a semi-direct product of a nilpotent Lie group $N$ and $A=\mathbb{R}^{+}$and, moreover, for a $H \in \mathcal{A}$ the Lie algebra of $A$ the eigenvalues of $\left.\operatorname{Ad}_{H}\right|_{N}$ are all greater than 0. Conversely, every such group equipped with a suitable left-invariant metric becomes a homogeneous Riemannian manifold with negative curvature. 
On $S$ we consider a second order left-invariant operator

$$
\mathcal{L}=\sum_{j=0}^{m} Y_{j}^{2}+Y
$$

such that $Y_{0}, \ldots, Y_{m}$ generate $\mathcal{S}$. Let $\pi: S \longrightarrow A=S / N$ be the canonical homomorphism. $d \pi(\mathcal{L})$ is a second order invariant operator on $\mathbb{R}^{+}$, hence

$$
d \pi(\mathcal{L})=\left(a \partial_{a}\right)^{2}-\gamma a \partial_{a}
$$

for a $\gamma \in \mathbb{R}$. $-\gamma a \partial_{a}$ is the $\mathcal{A}$-component of $Y$ and $\mathcal{L}=\mathcal{L}_{\gamma}$ is coercive, if and only if $\gamma \neq 0$.

Let $\mu_{t}$ be the semigroup of measures generated by $\mathcal{L}_{\gamma}$. If $\gamma \geq 0$, then there is a unique (up to a constant) positive Radon measure $\nu_{\gamma}$ on $N$ such that

$$
\check{\mu}_{t}^{\gamma} * \nu_{\gamma}=\nu_{\gamma}, \quad t>0
$$

[E]. For $\gamma>0$ the measure $\nu_{\gamma}$ is bounded, while $\nu_{0}$ is unbounded. The measures $\nu_{\gamma}, \gamma>0$ have been studied in various contexts [B], [E], [G], [Ra], see also [D1], [D2], [DH2], [DHZ]. In particular, the bounded $\mathcal{L}_{\gamma^{-}}$ harmonic functions, $\gamma>0$ are described as $\nu_{\gamma}$-Poisson integrals [Ra], [D1], [DH2] of $L^{\infty}$-functions on $N$. If $\gamma=0$, the only bounded $\mathcal{L}$ harmonic functions are constants but the unbounded measure $\nu_{0}$ gives rise to non-trivial positive $\mathcal{L}_{0}$ harmonic functions.

Also $\nu_{\gamma}$ plays an essential role in description of the Martin boundary for $\mathcal{L}_{\gamma}$ (and $\mathcal{L}_{-\gamma}$ ) both in the coercive and the noncoercive case. However, while the first case can be deduced from Ancona's theory [D2], the latter requires new methods. This is the main topic of our study here.

We make use of a probabilistic method introduced in [DH1] and continued in [DHZ]. The essence of it is a decomposition of the diffusion on $S$ generated by $a^{-2} \mathcal{L}$ into the "vertical component" generated by $\left(\partial_{a}\right)^{2}-(\gamma / a) \partial_{a}$ (Bessel process) and the "horizontal component" for which the transition probabilities conditioned on a trajectory $a_{t}$ of the "vertical component" satisfy some evolution equation (Chapter 3). The idea of this decomposition is very intuitive and goes back to $[\mathrm{M}],[\mathrm{MM}]$, cf. also $[\mathrm{K}],[\mathrm{S}]$, [Tay]. The available proofs of the properties of this decomposition are either very sketchy or quite involved. We give here a direct proof of it adapted to the situation of our interest.

The main aim of the present paper is to describe the Martin boundary for $\mathcal{L}_{\gamma}$, for all $\gamma \in \mathbb{R}$. In addition, we find lower and upper pointwise 
bounds for $\nu_{\gamma} . \nu_{\gamma}$ turns out to be the main building block for all minimal positive $\mathcal{L}_{\gamma}$.

In the simplest two dimensional case, i.e. when $S=$ " $a x+b$ " the description of the Martin boundary is due to Molchanov, [Mo]. Indeed, his technique is based on properties of the Bessel process, as is ours, only in the two-dimensional case the operator in the horizontal direction can be made independent of the vertical direction which makes the decomposition mentioned above superfluous, and all the arguments are much simpler.

\section{Preliminaries.}

Let

$$
\mathcal{S}=\mathcal{N} \oplus \mathcal{A}
$$

be a solvable Lie algebra which is the sum of its nilpotent ideal $\mathcal{N}$ and a one-dimensional algebra $\mathcal{A}=\mathbb{R}^{+}$. We assume that

there exists $H \in \mathcal{A}$ such that the real parts

of the eigenvalues of $\operatorname{ad}_{H}: \mathcal{N} \longmapsto \mathcal{N}$ are positive.

Let $N, A, S$ be the connected and simply connected Lie groups whose Lie algebras are $\mathcal{N}, \mathcal{A}, \mathcal{S}$ respectively. Then $S=N A$ is a semi-direct product of $N$ and $A=\mathbb{R}^{+}$.

On $S$ we consider a second order left-invariant operator

$$
\mathcal{L}=\sum_{j=0}^{m} Y_{j}^{2}+Y
$$

such that $Y_{0}, \ldots, Y_{m}$ generate $\mathcal{S}$. It follows from elementary linear algebra that $Y_{0}, \ldots, Y_{m}$ can be chosen in the way that $Y_{1}(e), \ldots, Y_{m}(e) \in \mathcal{N}$.

The decomposition (1.0) is not unique, i.e. there is no canonical choice of $A$. We put $A=\exp \left\{t Y_{0}: t>0\right\}$ and assume with no loss of generality that the real parts of the eigenvalues of $\operatorname{ad}_{Y_{0}}$ are strictly positive. Moreover, multiplying $\mathcal{L}$ by a constant we may assume that the real parts of $\operatorname{ad}_{Y_{0}}$ are large. Decomposing $s \in S$ as $s=x a, x \in N$, 
$a=\exp (\log a)\left(Y_{0}\right)$, we write

$$
\begin{aligned}
\mathcal{L} f(x a)= & \mathcal{L}_{\gamma} f(x a) \\
= & \left(\left(a \partial_{a}\right)^{2}-\gamma a \partial_{a}\right) f(x a) \\
& +\left(\sum_{j=1}^{m} \Phi_{a}\left(X_{j}\right)^{2}+\Phi_{a}(X)\right) f(x a),
\end{aligned}
$$

where $\Phi_{a}=\operatorname{Ad}_{\exp (\log a) Y_{0}}$ and $X, X_{1}, \ldots, X_{m}$ are left-invariant vector fields on $N$ and $X_{1}, \ldots, X_{m}$ generate $\mathcal{N}$. We shall keep the subscript $\gamma$ in $\mathcal{L}$ in order to stress the role of the $\mathcal{A}$-component of $Y$.

(1.1) together with the assumption on the length of $Y_{0}$ imply (see e.g. [DHZ]) that there are $m_{1}, m_{2}>2$ and $C>0$ such that

$$
\left\|\Phi_{a}\right\|_{\mathcal{N} \rightarrow \mathcal{N}} \leq C\left(a^{m_{1}}+a^{m_{2}}\right), \quad a>0
$$

In $N$ we define a "homogeneous" norm $|\cdot|$. Let $(\cdot, \cdot)$ be an arbitrary fixed inner product in $\mathcal{N}$ and let

$$
\langle X, Y\rangle=\int_{0}^{1}\left(\Phi_{a}(X), \Phi_{a}(Y)\right) \frac{d a}{a}, \quad\|X\|=\sqrt{\langle X, X\rangle} .
$$

We put

$$
|\exp X|=|X|=\left(\inf \left\{a>0:\left\|\Phi_{a}(X)\right\| \geq 1\right\}\right)^{-1} .
$$

Since for $X \neq 0$

$$
\begin{aligned}
& \lim _{a \rightarrow 0}\left\|\Phi_{a}(X)\right\|=0, \\
& \lim _{a \rightarrow \infty}\left\|\Phi_{a}(X)\right\|=\infty, \\
& \text { and } a \longrightarrow\left\|\Phi_{a}(X)\right\| \text { is increasing, }
\end{aligned}
$$

it follows that for every $Y \neq 0$ there is precisely one $a$ such that

$$
Y=\Phi_{a}(X), \quad|X|=1, \quad|Y|=a .
$$

If the action of $A$ on $N$ is diagonal, $|\cdot|$ is the usual homogeneous norm on $N$. Finally, let

$$
\sigma_{a}(\exp X)=\exp (\log a) Y_{0} \exp X \exp (-\log a) Y_{0}
$$


i.e. $\Phi_{a}$ is the differential of $\sigma_{a}$.

The space $\mathcal{H}_{b}$ of bounded harmonic functions for $\mathcal{L}$ is well known. If $\gamma \leq 0$, then bounded harmonic functions are constant. This is a consequence of $[\mathrm{BR}]$ ( $c f$. also [DH2]). If $\gamma>0, \mathcal{H}_{b}$ is in one-one correspondence with $L^{\infty}(N)$ via the Poisson integral

$$
F(s)=\int_{N} f(s \cdot x) m_{\gamma}(x) d x
$$

where $x \longrightarrow s \cdot x$ denotes the action of $S$ on $N=S / A$ ([Ra], [DH2]). $m_{\gamma}$ is a smooth, bounded positive function with $d \nu_{\gamma}(x)=m_{\gamma}(x) d x$ whence $\int_{N} m_{\gamma}(x) d x=1([\mathrm{D}])$. Moreover $[\mathrm{D}]$,

$$
C^{-1}(1+|x|)^{-Q-\gamma} \leq m_{\gamma}(x) \leq C(1+|x|)^{-Q-\gamma}, \quad x \in N .
$$

For $\gamma>0$ the function $m_{\gamma}$ is uniquely defined by two conditions

$$
\int_{N} m_{\gamma}(x) d x=1
$$

and

$$
P(x a)=a^{-Q} \breve{m}_{\gamma}\left(\sigma_{a^{-1}}(x)\right) \text { is } \mathcal{L} \text {-harmonic } .
$$

It turns out that the probability measure $m_{\gamma}$ is also the basic ingredient in the description of positive harmonic functions for all $\gamma \in$ $\mathbb{R}$.

Let

$$
Q=\operatorname{Re} \operatorname{Tr} \operatorname{ad}_{Y_{0}}
$$

and

$$
P_{y}(x a)=a^{-Q} \breve{m}_{\gamma}\left(\sigma_{a^{-1}}\left(y^{-1} x\right)\right)
$$

If $\gamma>0$, the family $\left\{P_{y}\right\}_{y \in N}$ and the function $a^{\gamma}$ are all the minimal positive $\mathcal{L}_{\gamma}$-harmonic functions ([A], cf also [D2]). The proofs (as well as the proof of (1.5)) are based on the Ancona's potential theory on manifolds with negative curvature. Since $\mathcal{L}_{-\gamma} f=a^{-\gamma} \mathcal{L}\left(a^{\gamma} f\right)$, the minimal positive $\mathcal{L}_{-\gamma^{-}}$-harmonic functions are 1 and $a^{-\gamma} P_{y}(x a)$.

The case $\gamma=0$ is essentially different, because Ancona's theory does not apply. To examine the Martin kernel we have to estimate the Green function $\mathcal{G}_{0}$ for $\mathcal{L}_{0}$ in another way. The final description of 
positive minimal $\mathcal{L}_{0}$-harmonic functions, however, is very similar to the case $\gamma \neq 0$.

Let $\mu_{t}$ be the semigroup of probability measures with the infinitesimal generator $\mathcal{L}_{0}$ and let $\mu=\mu_{1}$. The Markov chain on $N$ with the transition probability

$$
P(x, B)=\breve{\mu} * \delta_{x}(B), \quad x \in N, B \subset N,
$$

is a Harris chain with the unique (up to a multiplicative constant) positive Radon measure $\nu_{0}$ such that $\breve{\mu} * \nu_{0}=\nu_{0}$, [E]. $\nu_{0}$ has a smooth density $m_{0}$ which is not integrable in contrast to $m_{\gamma}, \gamma>0$.

The aim of this paper is to show

Theorem. The minimal positive $\mathcal{L}_{0}$-harmonic functions normalized at e are

the constant function 1

$$
\text { and } P_{y}(x a)=\frac{1}{m_{0}(y)} a^{-Q} \breve{m}_{0}\left(\sigma_{a^{-1}}\left(y^{-1} x\right)\right) \text {. }
$$

Moreover, we have

$$
C^{-1}(1+|x|)^{-Q} \leq m_{0}(x) \leq C(1+|x|)^{-Q}, \quad x \in N .
$$

To prove the theorem we proceed in the following way. For $\gamma=$ $-2 \alpha \leq 0$ we define a new operator

$$
L_{\gamma}=a^{-2} \mathcal{L}_{\gamma}
$$

which is not left-invariant on $S$. We study it on the space $N \times \mathbb{R}^{+}$. However, it has some homogeneity with respect to the family of "dilations" $D_{r}, r>0$ on $N \times \mathbb{R}^{+}$

$$
D_{r}(x, a)=\left(\sigma_{r}(x), r a\right)
$$

We have

$$
L_{\gamma}\left(f \cdot D_{r}\right)=r^{2} L_{\gamma} f \cdot D_{r}
$$

Also $L_{\gamma}$ commutes with the natural action of $N$ on $N \times \mathbb{R}^{+}$on the left. 
The Green function $G_{\gamma}$ for $L_{\gamma}$ is given by

$$
G_{\gamma}(x, a ; y, b)=\int_{0}^{\infty} p_{t}(x, a ; y, b) d t
$$

where

$$
T_{t} f(x a)=\int_{N \times \mathbb{R}^{+}} f(y, b) p_{t}(x, a ; y, b) b^{1+2 \alpha} d y d b
$$

is the heat semigroup on $L^{2}\left(a^{2 \alpha+1}\right)$ generated by $L_{\gamma}$ (see Theorem 5.6). By (1.10)

$$
p_{r^{2} t}(x, a ; y, b)=r^{-Q-2 \alpha-2} p_{t}\left(D_{r^{-1}}(x, a) ; D_{r^{-1}}(y, b)\right)
$$

and so

$$
G_{\gamma}(x, a ; y, b)=r^{-Q-2 \alpha} G_{\gamma}\left(D_{r^{-1}}(x, a) ; D_{r^{-1}}(y, b)\right)
$$

The operator $L_{\gamma}^{*}$ conjugate to

$$
L_{\gamma}=\partial_{a}^{2}+(1-\gamma) a^{-1} \partial_{a}+a^{-2} \sum_{j=1}^{m} \Phi_{a}\left(X_{j}\right)^{2}+a^{-2} \Phi_{a}(X),
$$

with respect to the measure $a^{1+2 \alpha} d x d a$ is

$$
L_{\gamma}^{*}=\partial_{a}^{2}+(1-\gamma) a^{-1} \partial_{a}+a^{-2} \sum_{j=1}^{m} \Phi_{a}\left(X_{j}\right)^{2}-a^{-2} \Phi_{a}(X) .
$$

Clearly,

$$
p_{t}^{*}(x, a ; y, b)=p_{t}(y, b ; x, a)
$$

and

$$
G_{\gamma}^{*}(x, a ; y, b)=G_{\gamma}(y, b ; x, a) .
$$

Although the case $\gamma=0$ is the most interesting for us, we keep the assumption $\gamma \leq 0$ to stress that our method works for all those cases. In particular, we obtain new proofs of (1.5) and (1.7). (Again conjugating the operator by $a^{\gamma}$.)

Let $\mathcal{G}_{\gamma}$ be the Green function for $\mathcal{L}_{\gamma}, \gamma \leq 0$. $\mathcal{G}_{\gamma}$ is uniquely defined by the following two conditions

$$
\mathcal{L}_{\gamma} \mathcal{G}_{\gamma}(\cdot ; y b)=-\delta_{y b}, \quad \text { as distributions }
$$


(Functions are identified with distributions via the right Haar measure $\left.a^{-1} d a d x.\right)$

$$
\text { For every } y b \in S, \mathcal{G}_{\gamma}(\cdot, y b) \text { is a potential for } \mathcal{L}_{\gamma} \text {. }
$$

It turns out that

$$
G_{\gamma}(x, a ; y, b) b^{-\gamma}=\mathcal{G}_{\gamma}(x a ; y b)
$$

Since the notions of potentials for $L_{\gamma}$ and $\mathcal{L}_{\gamma}$ coincide, the only condition to check is (1.15). By Theorem (5.6) we have

$$
\int G_{\gamma}(x, a ; y, b) L_{\gamma}^{*} \phi(x, a) a^{2 \alpha+1} d a d x=-\phi(y, b) .
$$

But

$$
\begin{aligned}
\int G_{\gamma}(x, a ; y, b) L_{\gamma}^{*} \phi(x, a) a^{2 \alpha+1} d a d x & \\
& =\int G_{\gamma}(x, a ; y, b) a^{2-\gamma} L_{\gamma}^{*} \phi(x, a) a^{-1} d a d x \\
& =\int G_{\gamma}(x, a ; y, b) a^{-\gamma} \mathcal{L}_{\gamma}^{*} \phi(x, a) a^{-1} d a d x
\end{aligned}
$$

which shows (1.17).

Using (1.17) we describe the Martin boundary for $\mathcal{L}_{0}$ (Theorem 6.3). The case $\gamma \neq 0$ was described in [D2]. For that we heavily use (1.13) to find appropriate estimates for Martin kernels.

(1.11) can be extended to $b=0$ (see Lemma (5.2) and (5.5)) as the limit of $G_{\gamma}\left(x, a ; y, b_{n}\right), b_{n} \longrightarrow 0$. More precisely,

$$
G_{\gamma}(x, a ; y, 0)=\lim _{b_{n} \rightarrow 0} G_{\gamma}\left(x, a ; y, b_{n}\right)
$$

as Radon measures. Then

$$
\breve{m}_{\gamma}(x)=G_{-\gamma}(x, 1 ; e, 0), \quad \gamma \geq 0 .
$$

(1.18) follows from the fact that

$$
G_{-\gamma}(x, a ; e, 0)=a^{-Q-2 \alpha} G_{-\gamma}\left(\sigma_{a^{-1}}(x), 1 ; e, 0\right)
$$


is $L_{-\gamma}$-harmonic. Hence $a^{-Q-2 \alpha} \breve{m}_{\gamma}\left(\sigma_{a^{-1}}(x)\right)$ is $\mathcal{L}_{-\gamma}$-harmonic, and so $a^{-Q} \breve{m}_{\gamma}\left(\sigma_{a^{-1}}(x)\right)$ is $\mathcal{L}_{\gamma^{-}}$-harmonic. But the last condition implies that for every $t$

$$
\breve{\mu}_{t} * m_{\gamma}=m_{\gamma}, \quad \gamma \geq 0,
$$

which uniquely determines $m_{\gamma}$.

Hence, from estimates on $G$ we conclude estimates for $m_{\gamma}$.

\section{Bessel Process.}

Let $b_{\alpha}(t)$ denotes the Bessel process with a parameter $\alpha \geq 0$, [RY], i.e. a continuous Markov process with state space $[0,+\infty)$ generated by $\Delta=\partial_{a}^{2}+(2 \alpha+1 / a) \partial_{a}, \alpha \geq 0$.

The transition function with respect to the measure $y^{2 \alpha+1} d y$ is given by $([\mathrm{RY}])$

$$
p_{t}(x, y)
$$

$$
= \begin{cases}c(\alpha) \frac{1}{2 t} \exp \left(\frac{-x^{2}-y^{2}}{4 t}\right) I_{\alpha}\left(\frac{x y}{2 t}\right) \frac{1}{(x y)^{\alpha}}, & \text { for } x, y>0 \\ c(\alpha)(2 t)^{-(\alpha+1)} \exp \left(\frac{-y^{2}}{4 t}\right), & \text { for } x=0, y>0\end{cases}
$$

where

$$
I_{\alpha}(x)=\sum_{k=0}^{\infty} \frac{\left(\frac{x}{2}\right)^{2 k+\alpha}}{k ! \Gamma(k+\alpha+1)}
$$

is the Bessel function [L]. Therefore, for $x \geq 0$ and $B \subset(0,+\infty)$

$$
\mathbf{P}_{x}\left(b_{\alpha}(t) \in B\right)=\int_{B} p_{t}(x, y) y^{2 \alpha+1} d y
$$

The Bessel process appears as the vertical component of the diffusion generated by $L_{\gamma}, \gamma=-2 \alpha$. The aim of this chapter is to recall the basic properties of the process $b_{\alpha}(t)$. The proofs are rather standard, we sketch them briefly for reader's convenience.

Lemma 2.2. Let $\Omega$ be the space of trajectories of the Bessel process $b_{\alpha}(t)$. For $b_{\alpha} \in \Omega$ and $\lambda>0$ define $\theta_{\lambda}\left(b_{\alpha}\right)(t)=\sqrt{\lambda} b_{\alpha}(t / \lambda)$. Assume that $b_{\alpha}(t)$ starts from $x$. Then: 
i) for every $\lambda>0, \widetilde{b}_{t}=\theta_{\lambda}\left(b_{\alpha}\right)(t)$ is the Bessel process (with a parameter $\alpha$ ) starting from $\sqrt{\lambda} x$,

ii) for every $\lambda>0, x \geq 0$,

$$
\mathbf{E}_{x} f \circ \theta_{\lambda}=\mathbf{E}_{\sqrt{\lambda} x} f .
$$

The Bessel process $b_{\alpha}$ on $\mathbb{R}^{+}$started at $x>0$ satisfies the following stochastic differential equation [RY, p. 416],

$$
b_{\alpha}(t)=x+\beta(t)+(2 \alpha+1) \int_{0}^{t} \frac{1}{b_{\alpha}(s)} d s,
$$

where $\beta(t)$ is the one-dimensional Brownian motion started at 0 . Consequently, we have

$$
\mathrm{P}_{x}\left[b_{\alpha}(s) \leq \lambda\right] \leq \mathrm{P}_{0}\left[b_{\alpha}(s) \leq \lambda\right] \quad \text { and } \quad \mathrm{P}_{x}[b(s) \leq \lambda] \leq \mathrm{P}_{x}[\beta(s) \leq \lambda] .
$$

Also, by the comparison theorem [RY, p. 364],

$$
\alpha \leq \alpha^{\prime} \text { then for all } s \geq 0, b_{\alpha}(s) \leq b_{\alpha^{\prime}}(s), \quad \text { almost everywhere, }
$$

whence

$$
b_{\alpha}(s) \leq\left|\beta_{n}(s)\right|, \quad \text { where } n=[2 \alpha]+3,
$$

and $\beta_{n}$ is the $n$-dimensional Brownian motion.

\section{Lemma 2.3.}

$$
\mathrm{P}_{a}\left[\max _{0 \leq s \leq t} \beta_{\alpha}(s) \leq \lambda\right] \leq e^{-\varepsilon\left(t / \lambda^{2}\right)}
$$

Indeed, Let $q=\mathrm{P}_{0}\left[\beta_{\alpha}(1) \leq 1\right]$. Then $q<1$ and

$$
\begin{aligned}
\mathrm{P}_{a}\left[\max _{0 \leq s \leq t} b_{\alpha}(s) \leq \lambda\right] & \leq \mathrm{P}_{a / \lambda}\left[\max _{0 \leq s \leq t / \lambda^{2}} b_{\alpha}(s) \leq 1\right] \\
& \leq \mathrm{E}_{0} \prod_{k=0}^{\left[t / \lambda^{2}\right]} \mathrm{P}_{b_{\alpha}(k)}\left[b_{\alpha}(1) \leq 1\right] \\
& \leq q^{\left[t / \lambda^{2}\right]} \\
& \leq e^{-\varepsilon\left(t / \lambda^{2}\right)}
\end{aligned}
$$


Lemma 2.4. There exist constants $c_{1}, c_{2}$ such that for every $R>0$ and for every $t>0$,

$$
\mathbf{P}_{R}\left(\inf _{s \in[0, t]} b_{\alpha}(s)<\frac{R}{2}\right) \leq c_{1} e^{-c_{2} R^{2} / t} .
$$

Indeed,

$$
\mathrm{P}_{R}\left[\inf _{s \in[0, t]} b_{\alpha}(s)<\frac{R}{2}\right] \leq \mathrm{P}_{R}\left[\inf _{s \in[0, t]} \beta(s)<\frac{R}{2}\right] \leq c_{1} e^{-c_{2} R^{2} / t} .
$$

Lemma 2.5. There exist constants $c_{1}, c_{2}$ such that for every $x \geq 0$, for every $\lambda>0$ and for every $t>0$,

$$
\mathbf{P}_{x}\left(\sup _{s \in[0, t]} b_{\alpha}(s)>x+\lambda\right) \leq c_{1} e^{-c_{2} \lambda^{2} / t} .
$$

Indeed, for $n=[2 \alpha]+3$

$$
\mathbf{P}_{x}\left(\sup _{s \in[0, t]} b_{\alpha}(s)>x+\lambda\right) \leq \mathbf{P}_{x}\left(\sup _{s \in[0, t]} \beta_{n}(s)>x+\lambda\right) \leq c_{1} e^{-c_{2} \lambda^{2} / t} .
$$

Lemma 2.6. Let $\xi>0$. There are constants $\delta, c_{1}, c_{2}>0$ such that for every $a \geq 0$ and $A>0$,

$$
\mathbf{P}_{a}\left(\int_{0}^{1} b_{\alpha}^{\xi}(s) d s<A\right) \leq c_{1} e^{-c_{2} A^{-\delta}} .
$$

Proof. Given positive $\delta$, we have

$$
\begin{aligned}
\mathbf{P}_{a}\left(\int_{0}^{1} b^{\xi}(s)\right. & d s<A) \\
\leq & \mathbf{P}_{a}\left(\sup _{s \in[0,1]} b_{\alpha}(s) \leq 2 A^{\delta}\right) \\
& +\mathbf{P}_{a}\left(\sup _{s \in[0,1]} b_{\alpha}(s)>2 A^{\delta},\left|\left\{s: b_{\alpha}(s)>A^{\delta}\right\}\right|<A^{1-\delta \xi}\right) .
\end{aligned}
$$

By Lemma 2.3,

$$
\mathbf{P}_{a}\left(\sup _{s \in[0,1]} b_{\alpha}(s) \leq 2 A^{\delta}\right) \leq c_{1} e^{-c_{2} A^{-\delta}} .
$$


To estimate the probability of

$$
\Omega=\left\{\sup _{s \in[0,1]} b_{\alpha}(s)>2 A^{\delta},\left|\left\{s: b_{\alpha}(s)>A^{\delta}\right\}\right|<A^{1-\delta \xi}\right\},
$$

we define the stopping time $\tau=\inf \left\{s: b_{\alpha}(s)=2 A^{\delta}\right\}$. Then by Lemma 2.4 ,

$$
\mathbf{P}_{a}(\Omega) \leq \mathbf{E}_{a} \mathbf{P}_{b_{\alpha}(\tau)}\left(\inf _{s \in\left[0, A^{1-\delta \xi}\right]} b_{\alpha}(s)<\frac{b_{\alpha}(0)}{2}\right) \leq c_{1} e^{-c_{2} A^{2 \delta-1+\delta \xi}} .
$$

We choose $\delta$ such that $2 \delta-1+\delta \xi<0$.

Corollary 2.7. Let $\xi \geq 0$. Then

$$
\sup _{a \geq 0} \mathbf{E}_{a}\left(\int_{0}^{1} b_{\alpha}^{\xi}(s) d s\right)^{-D / 2}<+\infty
$$

Proof. Since by the previous Lemma

$$
\mathbf{P}_{a}\left(\frac{1}{n+1} \leq \int_{0}^{1} b_{\alpha}^{\xi}(s) d s \leq \frac{1}{n}\right) \leq c_{1}^{-c_{2} n^{\delta}},
$$

we have

$$
\mathbf{E}_{a}\left(\int_{0}^{1} b_{\alpha}^{\xi}(s) d s\right)^{-D / 2} \leq \sum_{n}(n+1)^{D / 2} e^{-c_{2} n^{\delta}}<+\infty .
$$

\section{Solution of a heat equation on the product $N \times \mathbb{R}^{+}$.}

In this chapter we give an analytic proof of the decomposition of the diffusion on $N \times \mathbb{R}^{+}$into its components. Using it we find a convenient formula for the solution of the heat equation

$$
\left(L_{\gamma}-\partial_{t}\right) u(t, x, a)=0 \text {. }
$$

For a multi-index $\beta=\left(\beta_{1}, \ldots, \beta_{k}\right), \beta_{j} \in \mathbb{Z}^{+}$and a basis $X_{1}, \ldots, X_{n}$ of the Lie algebra $\mathcal{N}$ of the Lie group $N$ we write

$$
X^{\beta}=X_{1}^{\beta_{1}} \cdots X_{n}^{\beta_{n}}
$$


For $k=0,1, \ldots, \infty$ we define

$$
C^{k}=\left\{f: X^{\beta} f \in C(N), \text { for }|\beta|<k+1\right\}
$$

and

$$
C_{\infty}^{k}=\left\{f \in C^{k}: \lim _{x \rightarrow \infty} X^{\beta} f(x) \text { exists for }|\beta|<k+1\right\}
$$

For $k<\infty$ the space $C_{\infty}^{k}$ is a Banach space with the norm

$$
\|f\|_{C_{\infty}^{k}}=\sum_{|\beta| \leq k}\left\|X^{\beta} f\right\|_{C(N)}
$$

Let

$$
L_{\sigma(t)}=\sigma(t)^{-2}\left(\sum\left(\Phi_{\sigma(t)}\left(X_{j}\right)\right)^{2}+\Phi_{\sigma(t)}(X)\right) .
$$

For a continuous function $\sigma:[0,+\infty) \longrightarrow[0,+\infty)=A$ let $\left\{U^{\sigma}(s, t)\right.$, $0<s<t\}$ be the (unique) family of bounded operators on $C_{\infty}=C_{\infty}^{0}$ which satisfies

i) $U^{\sigma}(s, s)=I$,

ii) $U^{\sigma}(s, r) U^{\sigma}(r, t)=U^{\sigma}(s, t), s<r<t$,

iii) $\partial_{s} U^{\sigma}(s, t) f=-L_{\sigma(s)} U^{\sigma}(s, t) f$, for every $f \in C_{\infty}$,

iv) $\partial_{t} U^{\sigma}(s, t) f=U^{\sigma}(s, t) L_{\sigma(t)} f$ for every $f \in C_{\infty}$,

v) $U^{\sigma}(s, t): C_{\infty}^{2} \longrightarrow C_{\infty}^{2}$.

$U^{\sigma}(s, t)$ is a convolution operator $U^{\sigma}(s, t) f=f * p^{\sigma}(t, s)$, where $p^{\sigma}(t, s)$ is a probability measure with a smooth density. By ii) we have $p^{\sigma}(t, r) * p^{\sigma}(r, s)=p^{\sigma}(t, s)$ for $t>r>s$. Existence of $U^{\sigma}(s, t)$ follows from $[\mathrm{T}]$.

Let $d \mathbf{W}_{a}$ be the probability measure on the space $C\left([0,+\infty), \mathbb{R}^{+}\right)$, for the Bessel process $b_{\alpha}(t)=b_{t}$.

For $f \in C_{c}^{\infty}(N)$ we define

$$
\begin{aligned}
u(t, x, a) & =\int U^{\sigma}(0, t) f(x, \sigma(t)) d \mathbf{W}_{a}(\sigma) \\
& =\mathbf{E}_{a} U^{\sigma}(0, t) f(x, \sigma(t))
\end{aligned}
$$


Theorem 3.1. Let $\gamma=-2 \alpha$ and let $u=u(t, x, a)$ be the function on $N$ defined by (3.1). Then

$$
L_{\gamma} u(t, x, a)=\partial_{t} u(t, x, a), \quad \text { on } \mathbb{R}^{+} \times N \times \mathbb{R}^{+} .
$$

$u$ is continuous and

$$
u(0, x, a)=f(x, a), \quad \text { when } t \longrightarrow 0
$$

ProOF. First, we prove that $u=u(t, x, a)$ defined in (3.1) is a solution of the integral equation

$$
u(t, x, a)=\mathbf{E}_{a} f\left(x, b_{t}\right)+\int_{0}^{t} \mathbf{E}_{a} L\left(b_{t-s}\right) u\left(s, x, b_{t-s}\right) d s .
$$

To do this we observe that $\mathbf{E}_{a} L\left(b_{t-s}\right) u\left(s, x, b_{t-s}\right)$ is finite. Let $Y_{1}$, $\ldots, Y_{n}$ be a fixed basis of $\mathcal{N}$. Then

$$
\Phi_{a} X_{j}=\alpha_{1}^{j}(a) Y_{1}+\cdots+\alpha_{n}^{j}(a) Y_{n}
$$

where $\alpha_{i}^{j}$ 's are continuous functions and $\left|\alpha_{i}^{j}(a)\right| \leq C\left(a^{m_{1}}+a^{m_{2}}\right)$. Moreover,

$$
Y_{k} \int f *_{N} p^{\sigma}(s, 0)\left(x, \sigma_{s}\right) d \mathbf{W}_{a}(\sigma)
$$

and

$$
Y_{k} Y_{l} \int f *_{N} p^{\sigma}(s, 0)\left(x, \sigma_{s}\right) d \mathbf{W}_{a}(\sigma)
$$

are bounded for $x$ in a compact set. We have

$$
\begin{aligned}
L(a) u(s, x, a) & \\
= & L(a) \int U^{\sigma}(0, s) f\left(x, \sigma_{s}\right) d \mathbf{W}_{a}(\sigma) \\
= & L(a) \int f *_{N} p^{\sigma}(s, 0)\left(x, \sigma_{s}\right) d \mathbf{W}_{a}(\sigma) \\
= & a^{-2} \sum_{j, k, l} \alpha_{k}^{j}(a) \alpha_{l}^{j}(a) Y_{k} Y_{l} \int f *_{N} p^{\sigma}(s, 0)\left(x, \sigma_{s}\right) d \mathbf{W}_{a}(\sigma) \\
& +a^{-2} \sum_{j, k} \alpha_{k}^{j}(a) Y_{k} \int f *_{N} p^{\sigma}(s, 0)\left(x, \sigma_{s}\right) d \mathbf{W}_{a}(\sigma)
\end{aligned}
$$


and, by the above remarks

$$
|L(a) u(s, x, a)| \leq C\left(a^{m_{3}}+a^{m_{4}}\right),
$$

where

$$
m_{3}=\min \left\{m_{1}, m_{2}, 2 m_{1}, 2 m_{2}, m_{1}+m_{2}\right\}-2>0
$$

and

$$
m_{4}=\max \left\{m_{1}, m_{2}, 2 m_{1}, 2 m_{2}, m_{1}+m_{2}\right\}-2 .
$$

It follows that $\mathbf{E}_{a} L\left(b_{t-s}\right) u\left(s, x, b_{t-s}\right)$ is finite. Indeed, by (3.4) and (3.5), proceeding as before (i.e. replacing $a$ by $b_{t-s}$ ) we obtain

$$
\left|\mathbf{E}_{a} L\left(b_{t-s}\right) u\left(s, x, b_{t-s}\right)\right| \leq C \mathbf{E}_{a}\left(b_{t-s}^{m_{3}}+b_{t-s}^{m_{4}}\right) .
$$

Now we calculate

$$
\begin{aligned}
\mathbf{E}_{a} L\left(b_{t-s}\right) u(s, & \left.x, b_{t-s}\right) \\
& =\int L\left(b_{t-s}\right) u\left(s, x, b_{t-s}\right) d \mathbf{W}_{a}(b) \\
& =\int L\left(b_{t-s}\right) \int U^{\sigma}(0, s) f\left(x, \sigma_{s}\right) d \mathbf{W}_{b_{t-s}}(\sigma) d \mathbf{W}_{a}(b) \\
& =\iint L\left(b_{t-s}\right) U^{\sigma}(0, s) f\left(x, \sigma_{s}\right) d \mathbf{W}_{b_{t-s}}(\sigma) d \mathbf{W}_{a}(b) \\
& =\int L\left(b_{t-s}\right) U^{b}(t-s, t) f\left(x, b_{t}\right) d \mathbf{W}_{a}(b) .
\end{aligned}
$$

By (3.6), and the Fubini's theorem we obtain

$$
\begin{aligned}
\int_{0}^{t} \mathbf{E}_{a} L\left(b_{t-s}\right) u(s, x & \left., b_{t-s}\right) d s \\
& =\iint_{0}^{t} L\left(b_{t-s}\right) U^{b}(t-s, t) f\left(x, b_{t}\right) d s d \mathbf{W}_{a}(b)
\end{aligned}
$$

but

$$
\int_{0}^{t} L\left(b_{t-s}\right) U^{b}(t-s, t) f\left(x, b_{t}\right) d s=U^{b}(0, t) f\left(x, b_{t}\right)-f\left(x, b_{t}\right) .
$$


Indeed by iii) we get

$$
\begin{aligned}
\frac{d}{d s} U^{b}(t-s, t) f\left(x, b_{t}\right) & =-\left.\frac{d}{d s} U^{b}(\cdot, t) f\left(x, b_{t}\right)\right|_{t-s} \\
& =-\left(-L\left(b_{t-s}\right) U^{b}(t-s, t) f\left(x, b_{t}\right)\right) \\
& =L\left(b_{t-s}\right) U^{b}(t-s, t) f\left(x, b_{t}\right)
\end{aligned}
$$

Therefore,

$$
\begin{aligned}
\int_{0}^{t} \mathbf{E}_{a} L\left(b_{t-s}\right) u(s, & \left.x, b_{t-s}\right) d s \\
& =\int U^{b}(0, t) f\left(x, b_{t}\right) d \mathbf{W}_{a}(b)-\int f\left(x, b_{t}\right) d \mathbf{W}_{a}(b) \\
& =u(t, x, a)-\mathbf{E}_{a} f\left(x, b_{t}\right) .
\end{aligned}
$$

Now we are going to prove that $u$ is a solution of the differential equation (3.2). Since $u$ is a solution of (3.3) we have

$$
\begin{aligned}
& \frac{u(t+h, x, a)-u(t, x, a)}{h} \\
& =\frac{\mathbf{E}_{a} f\left(x, b_{t+h}\right)-\mathbf{E}_{a} f\left(x, b_{t}\right)}{h}+\frac{1}{h} \int_{0}^{t}\left(\mathbf{E}_{a} L\left(b_{t+h-s}\right) u\left(s, x, b_{t+h-s}\right)\right. \\
& \left.\quad-\mathbf{E}_{a} L\left(b_{t-s}\right) u\left(s, x, b_{t-s}\right)\right) d s \\
& \quad+\frac{1}{h} \int_{t}^{t+h} \mathbf{E}_{a} L\left(b_{t+h-s}\right) u\left(s, x, b_{t+h-s}\right) d s .
\end{aligned}
$$

Let $\Delta$ be the infinitesimal generator of the Bessel process i.e.

$$
\Delta=\partial_{a}^{2}+\frac{2 \alpha+1}{a} \partial_{a} .
$$

Letting $h$ to 0 we get

$$
\begin{aligned}
& \partial_{t} u(t, x, a) \\
& \quad=\Delta \mathbf{E}_{a} f\left(x, b_{t}\right)+\Delta \int_{0}^{t} \mathbf{E}_{a} L\left(b_{t-s}\right) u\left(s, x, b_{t-s}\right) d s+L(a) u(t, x, a)
\end{aligned}
$$

in a sense of distributions. 
On the other hand, since $u$ is a solution of (3.3) thus $L u(t, x, a)$

$$
\begin{aligned}
& =(L(a)+\Delta) u(t, x, a) \\
& =L(a) u(t, x, a)+\Delta\left(\mathbf{E}_{a} f\left(x, b_{t}\right)+\int_{0}^{t} \mathbf{E}_{a} L\left(b_{t-s}\right) u\left(s, x, b_{t-s}\right) d s\right) \\
& =L(a) u(t, x, a)+\Delta \mathbf{E}_{a} f\left(x, b_{t}\right)+\Delta \int_{0}^{t} \mathbf{E}_{a} L\left(b_{t-s}\right) u\left(s, x, b_{t-s}\right) d s .
\end{aligned}
$$

So $u$ is a solution of (3.2).

Theorem 3.2. Let

$$
T_{t} f(x, a)=\int U^{\sigma}(0, t) f\left(x, \sigma_{t}\right) d \mathbf{W}_{a}(\sigma) .
$$

Then $\left\{T_{t}\right\}$ is a semigroup.

PROOF.

$$
\begin{aligned}
T_{s}\left(T_{t} f\right)(x, a) & =\int U^{b}(0, s) T_{t} f\left(x, b_{s}\right) d \mathbf{W}_{a}(b) \\
& =\int U^{b}(0, s) \int U^{\sigma}(0, t) f\left(x, \sigma_{t}\right) d \mathbf{W}_{b_{s}}(\sigma) d \mathbf{W}_{a}(b) \\
& =\int U^{b}(0, s) U^{b}(s, s+t) f\left(x, b_{s+t}\right) d \mathbf{W}_{a}(b) \\
& =\int U^{b}(0, s+t) f\left(x, b_{s+t}\right) d \mathbf{W}_{a}(b) \\
& =T_{s+t} f(x, a)
\end{aligned}
$$

where in the third equality we have used the Markov property.

\section{Estimate of the evolution kernels by the Nash inequality.}

Let $X, X_{1}, \ldots, X_{m}$ be as in (1.2),

$$
\begin{gathered}
L_{a}=a^{-2}\left(\sum_{j=1}^{m}\left(\Phi_{a} X_{j}\right)^{2}+\Phi_{a}(X)\right), \\
\Delta_{0}=\sum_{j=1}^{m} X_{j}^{2},
\end{gathered}
$$


and

$$
\Delta=\Delta_{0}+X .
$$

Let $\sigma:[0,+\infty) \longrightarrow[0,+\infty)$ be a continuous function such that $\sigma(t)>0$ for $t>0$, and $p^{\sigma}(t, s, x)=p^{\sigma}(t, s)(x), s<t$ be the evolution generated by the operator $L_{\sigma(t)}+\partial_{t}$.

The aim of this Chapter is to prove the following estimate for $p^{\sigma}(t, 0, x)$ :

Theorem 4.1. For every compact set $K \subset N$, which does not contain the identity element e of $N$, there exist positive constants $C_{1}, C_{2}, m_{3}$, $m_{4}$ and $n \leq Q$ such that for every $x \in K$ and for every $t$,

$$
p^{\sigma}(t, 0, x) \leq C_{1}\left(\int_{0}^{t} \sigma^{-2(1-Q / n)}(u) d u\right)^{-n / 2} \exp \left(-\frac{C_{2}}{A(0, t)}\right)
$$

where

$$
A(s, t)=\int_{s}^{t}\left(\sigma^{m_{3}}(u)+\sigma^{m_{4}}(u)\right) d u
$$

The main tool in the proof of the above theorem is the Nash inequality (see e.g. [VSC])

$$
\|f\|_{L^{2}}^{2+4 / n} \leq-C(\Delta f, f)\|f\|_{L^{1}}^{4 / n}=\left(\Delta_{0} f, f\right)\|f\|_{L^{1}}^{4 / n}
$$

for all $f \in C_{0}^{\infty}(N)$, where $d$ is the local dimension of $\left(N, X_{1}, \ldots, X_{m}\right)$ and $D$ is the dimension at infinity of $\left(N, X_{1}, \ldots, X_{m}\right) n$ is any number satisfying $d \leq n \leq D$ (see [VSC]). Let $Q_{t}$ be the heat semi-group generated by $\Delta_{0}$. Then

$$
\left\|Q_{t}\right\|_{L^{1} \rightarrow L^{\infty}} \leq C \begin{cases}t^{-d / 2}, & \text { if } t \leq 1 \\ t^{-D / 2}, & \text { if } t \geq 1\end{cases}
$$

(Theorem IV.4.1 in [VSC]) and so (4.1) follows by the Nash theorem (Theorem II.5.2 in [VSC]). Since we can make $Q$ arbitrarily big (see $1.6), \xi=-2(1-Q / n)$ is positive.

Proof of Theorem 4.1. We start with some integral estimates on $f * p^{\sigma}(t, s)$.

Let $0 \leq \varphi \in C_{c}^{\infty}(N)$, supp $\varphi \subset B_{r}(e)$ and $\int \varphi=1$ ( $r$ will be fixed later). Let $\eta(x)=\tau * \varphi(x)$ where $\tau$ is a left invariant Riemannian metric 
on $N$. There exists a positive constant $C$ such that if $Y_{1}, \ldots, Y_{n}$ is a fixed basis of $\mathcal{N}$ then

$$
\left|Y_{j} \eta(x)\right| \leq C, \quad\left|Y_{i} Y_{j} \eta(x)\right| \leq C, \quad \text { for } i, j=1, \ldots, n
$$

[H]. Moreover,

$$
\tau(x) \leq \int\left(\tau\left(x y^{-1}\right)+\tau(y)\right) \varphi(y) d y \leq \eta(x)+r,
$$

and

$$
\eta(e)=\int \tau\left(y^{-1}\right) \varphi(y) d y \leq r
$$

For a natural number $m$ let $\eta_{m}(x)=\tau_{m} * \varphi(x)$, where

$$
\tau_{m}(x)=\min \{m, \tau(x)\} .
$$

Then there exists a positive constant $C$ such that for every $m,(4.3)$, (4.4) and (4.5) hold with $\eta_{m}$ and $\tau_{m}$ instead of $\eta$ and $\tau$ respectively.

We have

$$
\left(\partial_{s}\left(f * p^{\sigma}(t, s), e^{\alpha \eta_{m}}\right)=-\left(f * p^{\sigma}(t, s), L_{\sigma(s)}^{*} e^{\alpha \eta_{m}}\right)\right)
$$

(4.6) is obvious, if instead of $e^{\alpha \eta_{m}}$ we put $e^{\alpha \eta_{m}} \psi$, where $\psi \in C_{0}^{\infty}(N)$. So to conclude (4.6) we take the sequence $\psi_{j}=\psi \circ \sigma_{a_{j}}$ for $\psi \in C_{0}^{\infty}(N)$ such that $\psi(0)=1$ and $a_{j} \longrightarrow 0$. Since $\sigma_{a_{j}}(x) \longrightarrow e$ for every $x \in N$ and, by (1.3), $\left|\Phi_{a_{j}}\left(X_{j}\right) \psi\right| \longrightarrow 0$, we obtain (4.6) as the limit of

$$
\partial_{s}\left(f * p^{\sigma}(t, s), e^{\alpha \eta_{m}} \psi_{j}\right)=-\left(f * p^{\sigma}(t, s), L_{\sigma(s)}^{*}\left(e^{\alpha \eta_{m}} \psi_{j}\right)\right) .
$$

Therefore, by (1.2) and (4.3),

$$
\begin{aligned}
\partial_{s}\left(f * p^{\sigma}(t, s)\right. & \left., e^{\alpha \eta_{m}}\right) \\
\leq & C\left(\alpha+\alpha^{2}\right) \sigma^{-2}(s)\left(\sigma^{m_{1}}(s)+\sigma^{m_{2}}(s)\right)^{2}\left(f * p^{\sigma}(t, s), e^{\alpha \eta_{m}}\right) \\
& +C \alpha \sigma^{-2}(s)\left(\sigma^{m_{1}}(s)+\sigma^{m_{2}}(s)\right)\left(f * p^{\sigma}(t, s), e^{\alpha \eta_{m}}\right) .
\end{aligned}
$$

Thus

$$
\frac{\partial_{s}\left(f * p^{\sigma}(t, s), e^{\alpha \eta_{m}}\right)}{\left(f * p^{\sigma}(t, s), e^{\alpha \eta_{m}}\right)} \leq C\left(\alpha+\alpha^{2}\right)\left(\sigma^{m_{3}}(s)+\sigma^{m_{4}}(s)\right)
$$


and so

$$
\left(f * p^{\sigma}(t, s), e^{\alpha \eta_{m}}\right) \leq\left(f, e^{\alpha \eta_{m}}\right) \exp \left(C\left(\alpha+\alpha^{2}\right) A(s, t)\right),
$$

where

$$
A(s, t)=\int_{s}^{t}\left(\sigma^{m_{3}}(u)+\sigma^{m_{4}}(u)\right)
$$

Therefore,

$$
\begin{aligned}
\left(p^{\sigma}(t, s), e^{\alpha \eta_{m}}\right) & \leq e^{\alpha \eta_{m}(e)} \exp \left(C\left(\alpha+\alpha^{2}\right) A(s, t)\right) \\
& \leq e^{\alpha r} \exp \left(C\left(\alpha+\alpha^{2}\right) A(s, t)\right)
\end{aligned}
$$

Now for $m \longrightarrow \infty(4.4)$ and (4.5) yield

$$
\begin{aligned}
\left(p^{\sigma}(t, s), e^{\alpha \tau}\right) & \leq\left(p^{\sigma}(t, s), e^{\alpha(\eta+r)}\right) \\
& \leq e^{2 \alpha r} \exp \left(C\left(\alpha+\alpha^{2}\right) A(s, t)\right)
\end{aligned}
$$

The next step is the Nash inequality for $L_{a}$. Applying (4.2) to $f \circ \sigma_{a}$ we obtain

$$
\begin{aligned}
a^{-Q(1+2 / n)}\|f\|_{L^{2}}^{2(1+2 / n)} & \leq-C a^{-Q}\left(a^{2} L_{a} f, f\right) a^{-4 Q / n}\|f\|_{L^{1}}^{4 / n} \\
& =-C a^{-Q+2-4 Q / n}\left(L_{a} f, f\right)\|f\|_{L^{1}}^{4 / n}
\end{aligned}
$$

Thus

$$
\|f\|_{L^{2}}^{2(1+2 / n)} \leq-C a^{2(1-Q / n)}\left(L_{a} f, f\right)\|f\|_{L^{1}}^{4 / n} .
$$

Now we proceed similarly as in the case of semigroups (e.g. [VSC]).

For a function $0 \leq f \in C_{c}^{\infty}(N)$ such that $\int f=1$ we define

$$
f_{s}(x)=f * p^{\sigma}(t, s)(x), \quad h_{s}(x)=\left\|f_{s}\right\|_{L^{2}}^{2} .
$$

Then

$$
\begin{aligned}
-\partial_{s} h_{s} & =-\partial_{s}\left(f_{s}, f_{s}\right) \\
& =2\left(L_{\sigma(s)} f_{s}, f_{s}\right) \\
& \leq-2 C^{-1} \sigma^{-2(1-Q / n)}(s)\left\|f_{s}\right\|_{L^{2}}^{2(1+2 / n)} \\
& =-C \sigma^{-2(1-Q / n)}(s) h_{s}^{1+2 / n}
\end{aligned}
$$


(By (4.7) we may exchange $\partial_{s}$ with the integral.) So

$$
-\partial_{s} h_{s} h_{s}^{-1-2 / n} \leq-C \sigma^{-2(1-Q / n)}(s) .
$$

Hence

$$
-\int_{s}^{t} \partial_{u} h_{u} h_{u}^{-1-2 / n} d u=\left.\frac{n}{2} h_{u}^{-2 / n}\right|_{u=s} ^{u=t} \leq-C \int_{s}^{t} \sigma^{-2(1-Q / n)}(u) d u .
$$

Thus

$$
\frac{n}{2}\left(h_{t}^{-2 / n}-h_{s}^{-2 / n}\right) \leq-C \int_{s}^{t} \sigma^{-2(1-Q / n)}(u) d u .
$$

Since $h_{t}^{-2 / n}>0$,

$$
-\frac{n}{2} h_{s}^{-2 / n} \leq-C \int_{s}^{t} \sigma^{-2(1-Q / n)}(u) d u
$$

and so

$$
\left\|f * p^{\sigma}(t, s)\right\|_{L^{2}}=h_{s}^{1 / 2} \leq C\left(\int_{s}^{t} \sigma^{-2(1-Q / n)}(u) d u\right)^{-n / 2}\|f\|_{L^{1}} .
$$

Therefore,

$$
\begin{aligned}
\left\|p^{\sigma}(t, s)\right\|_{L^{2}} \leq & C\left(\int_{s}^{t} \sigma^{-2(1-Q / n)}(u) d u\right)^{-n / 4} \\
\left\|p^{\sigma}(t, s)\right\|_{L^{\infty}} \leq & \left\|p^{\sigma}(t, u)\right\|_{L^{2}}\left\|p^{\sigma}(u, s)\right\|_{L^{2}} \\
\leq & C\left(\int_{\xi}^{t} \sigma^{-2(1-Q / n)}(u) d u\right)^{-n / 4} \\
& \cdot\left(\int_{s}^{\xi} \sigma^{-2(1-Q / n)}(u) d u\right)^{-n / 4} .
\end{aligned}
$$

Taking $\xi$ such that

$$
\begin{aligned}
\int_{s}^{\xi} \sigma^{-2(1-Q / n)}(u) d u & =\int_{\xi}^{t} \sigma^{-2(1-Q / n)}(u) d u \\
& =\frac{1}{2} \int_{s}^{t} \sigma^{-2(1-Q / n)}(u) d u
\end{aligned}
$$


we obtain

$$
\left\|p^{\sigma}(t, s)\right\|_{L^{\infty}} \leq C\left(\int_{s}^{t} \sigma^{-2(1-Q / n)}(u) d u\right)^{-n / 2} .
$$

By the subadditivity of the metric $\tau$, estimates (4.7) and (4.9) we have

$$
\begin{aligned}
p^{\sigma}(t, & 0, x) e^{\alpha \tau(x)} \\
\leq & \int p^{\sigma}(t, s, x) p^{\sigma}\left(s, 0, x y^{-1}\right) e^{\alpha \tau(y)} e^{\alpha \tau\left(x y^{-1}\right)} d y \\
\leq & \left\|p^{\sigma}(t, s)\right\|_{L^{\infty}}^{1 / 2}\left\|p^{\sigma}(s, 0)\right\|_{L^{\infty}}^{1 / 2}\left(p^{\sigma}(t, s), e^{2 \alpha \tau}\right)^{1 / 2}\left(p^{\sigma}(s, 0), e^{2 \alpha \tau}\right)^{1 / 2} \\
\leq & C\left(\int_{s}^{t} \sigma^{-2(1-Q / n)}(u) d u\right)^{-n / 4}\left(\int_{0}^{s} \sigma^{-2(1-Q / n)}(u) d u\right)^{-n / 4} \\
& \cdot e^{4 \alpha r} \exp \left(C\left(\alpha+\alpha^{2}\right) A(s, t)\right) \exp \left(C\left(\alpha+\alpha^{2}\right) A(0, s)\right) \\
= & C\left(\int_{s}^{t} \sigma^{-2(1-Q / n)}(u) d u\right)^{-n / 4}\left(\int_{0}^{s} \sigma^{-2(1-Q / n)}(u) d u\right)^{-n / 4} \\
& \cdot e^{4 \alpha r} \exp \left(C\left(\alpha+\alpha^{2}\right) A(0, t)\right) .
\end{aligned}
$$

Now for the $s$ such that in the last product the first two factors are equal we obtain

$$
\begin{aligned}
& p^{\sigma}(t, 0, x) e^{\alpha \tau(x)} \\
& \quad \leq C\left(\int_{0}^{t} \sigma^{-2(1-Q / n)}(u) d u\right)^{-n / 2} e^{4 \alpha r} \exp \left(C\left(\alpha+\alpha^{2}\right) A(0, t)\right) .
\end{aligned}
$$

If $\alpha=\varepsilon \tau(x) / A(0, t)$, then

$$
\begin{aligned}
p^{\sigma}(t, 0, x) \leq & C\left(\int_{0}^{t} \sigma^{-2(1-Q / n)}(u) d u\right)^{-n / 2} \\
& \cdot \exp \left(\frac{4 \varepsilon r \tau(x)}{A(0, t)}+C \varepsilon \tau(x)+\frac{C \varepsilon^{2} \tau^{2}(x)}{A(0, t)}-\frac{\varepsilon \tau^{2}(x)}{A(0, t)}\right) .
\end{aligned}
$$

Now our assumptions on $K$ imply that we may neglect $C \varepsilon \tau(x)$ and we can find $r$ such that $r<\tau(x) / 16, x \in K$. Moreover, we assume that $C \varepsilon<1 / 4$. Then

$$
p^{\sigma}(t, 0, x) \leq C\left(\int_{0}^{t} \sigma^{-2(1-Q / n)}(u) d u\right)^{-n / 2} \exp \left(\frac{-\varepsilon \tau^{2}(x)}{2 A(0, t)}\right)
$$


and the proof is completed.

Theorem 4.11. Assume that

$$
\lambda \leq \sigma(s) \leq \Lambda, \quad \text { for } s \in[r, r+T]
$$

Given $0<T_{1}<T_{2}<T$ and a neighborhood $B$ of e, we can find $C>0$ independent on $r$ such that

$$
p^{\sigma}(r, r+t) \geq C, \quad \text { for } z \in B, 0<T_{1} \leq t \leq T_{2}<T
$$

and any $\sigma$ satisfying (4.12).

Proof. Although we have an evolution here, not a semigroup, the proof of (4.12) is the same ([SS, p. 106-108]). It is based on the Poincaré inequality and upper bound estimates we have just proved. Let $\rho_{a}$ be the optimal control metric defined by the vector fields $a^{-2} \Phi_{a}\left(X_{1}\right), \ldots$, $a^{-2} \Phi_{a}\left(X_{m}\right)$ and let $B_{r, a}=\left\{x \in N: \rho_{a}(x)<r\right\}$. Then

$$
\begin{aligned}
\min _{z \in \mathbb{R}} \int_{B_{r, a}}|f(x)-z|^{2} d x & \leq \int_{B_{r, a}}\left|f(x)-f_{r, a}\right|^{2} d x \\
& \leq C r^{2} \int_{B_{(3 / 2) r, a}}|\nabla f(x)|^{2} d x,
\end{aligned}
$$

where,

$$
f_{r, a}=\frac{1}{\left|B_{r, a}\right|} \int_{B_{r, a}} f(y) d y \quad \text { and } \quad|\nabla f|^{2}=\sum_{j=1}^{m}\left(X_{j}\right)^{2} .
$$

The constant $C$ does not depend on $a, r$. (4.14) implies

$$
\begin{aligned}
\min _{z \in \mathbb{R}} \int|f(x)-z|^{2} \Psi_{a, r}(x) d x & =\int\left|f(x)-f_{\Psi_{r, a}}\right|^{2} \Psi_{a, r}(x) d x \\
& \leq C r^{2} \int|\nabla f(x)|^{2} \Psi_{a, 2 r}(x) d x
\end{aligned}
$$

where

$$
f_{\Psi_{a, r}}=\frac{\int f(y) \Psi_{a, r}(y) d y}{\int \Psi_{a, r}(y) d y}
$$


and

$$
\Psi_{a, r}(x)= \begin{cases}\left(\frac{1-\rho_{a}(x)}{r}\right)^{2}, & \text { if } \rho_{a}(x)<r, \\ 0, & \text { if } \rho_{a}(x) \geq r,\end{cases}
$$

and $c$ does not depend on $a$. Having (4.15) we follow the argument on [SS, p. 106-108].

\section{Green function for $L_{\gamma}$.}

Let

$$
T_{t} f(x, a)=\mathbf{E}_{a} U^{\sigma}(0, t) f\left(x, \sigma_{t}\right)
$$

be the semigroup of operators generated by $L_{\gamma}$. Since

$$
\left|\mathbf{E}_{a} U^{\sigma}(0, t) f\left(x, \sigma_{t}\right)\right| \leq\|f\|_{L^{\infty}} \text { and } \mathbf{E}_{a} U^{\sigma}(0, t) f\left(x, \sigma_{t}\right) \geq 0 \text { for } f \geq 0,
$$

for every $x \in N, a \geq 0, t>0$, there exists a probability measure $p_{t}(x, a ; \cdot, \cdot)$ such that

$$
T_{t} f(x, a)=\int_{N \times \mathbb{R}^{+}} f(y, b) p_{t}(x, a ; d y, d b) .
$$

Moreover, $p_{t}(x, a ; \cdot, \cdot) \in L^{2}\left(N \times \mathbb{R}^{+}, d x \otimes a^{2 \alpha+1} d a\right)$. Indeed,

$$
\left|U^{\sigma}(0, t) f(x, \sigma(t))\right| \leq\left\|p^{\sigma}(t, 0)\right\|_{L^{2}(d x)}\left(\int|f(x, \sigma(t))|^{2} d x\right)^{1 / 2} .
$$

Therefore,

$$
\begin{aligned}
\left|T_{t} f(x, a)\right| & \leq\left(\mathbf{E}_{a}\left\|p^{\sigma}(t, 0)\right\|_{L^{2}(d x)}^{2}\right)^{1 / 2}\left(\mathbf{E}_{a} \int|f(x, \sigma(t))|^{2} d x\right)^{1 / 2} \\
& \leq c(a, t)\left(\mathbf{E}_{a}\left\|p^{\sigma}(t, 0)\right\|_{L^{2}(d x)}^{2}\right)^{1 / 2}\|f\|_{L^{2}\left(d x \otimes a^{2 \alpha+1} d a\right)}
\end{aligned}
$$

because for a fixed $t$ the kernel (2.1) is bounded as a function of space variable. By (4.9), Lemma 2.2 and Corollary 2.15, $\mathbf{E}_{a}\left\|p^{\sigma}(t, 0)\right\|_{L^{2}(d x)}^{2}<$ $\infty$ and so, for every $t, x, a$,

$$
p_{t}(x, a ; \cdot, \cdot) \in L^{2}\left(N \times \mathbf{R}^{+}, d x \otimes d a^{2 \alpha+1} d a\right) .
$$

Now a standard argument shows that for fixed $x \in N, a>0$,

$$
\left(L^{*}-\partial_{t}\right) p \cdot(x, a ; \cdot, \cdot)=0 .
$$


We want to have (5.1) also for $a=0$.

Lemma 5.2. Given $f \in C_{c}^{\infty}\left(N \times \mathbb{R}^{+} \times \mathbb{R}^{+}\right)$, we have

$$
\begin{aligned}
\lim _{a \rightarrow 0} \int p_{t}(x, a ; y, b) & f(y, b, t) d y b^{2 \alpha+1} d b d t \\
= & \int p_{t}(x, 0 ; y, b) f(y, b, t) d y b^{2 \alpha+1} d b d t .
\end{aligned}
$$

Proof. We rewrite (5.3) as

$$
\lim _{a \rightarrow 0} \mathbf{E}_{a} U^{\sigma}(0, t) f(x, \sigma(t), t)=\mathbf{E}_{0} U^{\sigma}(0, t) f(x, \sigma(t), t) .
$$

Since the trajectories are continuous, it is enough to show that $U^{\sigma}(0, t) f(x, \sigma(t), t)$ is a continuous function of the trajectory $\sigma$. For an arbitrary fixed $T>0$ let

$$
d\left(\sigma, \sigma^{\prime}\right)=\sup _{t \in[0, T]}\left|\sigma(t)-\sigma^{\prime}(t)\right| .
$$

We have

$$
\begin{aligned}
& U^{\sigma}(s, t) f(x, \sigma(t), t)-U^{\sigma^{\prime}}(s, t) f(x, \sigma(t), t) \\
& =U^{\sigma}(s, t) f(x, \sigma(t), t)-U^{\sigma}(s, t) f\left(x, \sigma^{\prime}(t), t\right) \\
& \quad+U^{\sigma}(s, t) f\left(x, \sigma^{\prime}(t), t\right)-U^{\sigma^{\prime}}(s, t) f\left(x, \sigma^{\prime}(t), t\right)
\end{aligned}
$$

and

$$
\begin{aligned}
\mid U^{\sigma}(s, t) f(x, \sigma(t), t)-U^{\sigma}(s, t) & f\left(x, \sigma^{\prime}(t), t\right) \mid \\
& \leq \sup _{x, t}\left|f(x, \sigma(t), t)-f\left(x, \sigma^{\prime}(t), t\right)\right|,
\end{aligned}
$$

which clearly tends to 0 if $d\left(\sigma, \sigma^{\prime}\right) \longrightarrow 0$. The second term in (5.4) can be written as

$$
\begin{aligned}
& U^{\sigma}(s, t) f\left(x, \sigma^{\prime}(t), t\right)-U^{\sigma^{\prime}}(s, t) f\left(x, \sigma^{\prime}(t), t\right) \\
&=\int_{s}^{t} U^{\sigma}(s, r)\left(L\left(\sigma_{r}\right)-L\left(\sigma_{r}^{\prime}\right)\right) U^{\sigma^{\prime}}(r, t) f\left(x, \sigma^{\prime}(t), t\right) d r .
\end{aligned}
$$

It also tends to 0 , because for $\xi \geq 0$

$$
\lim _{\sigma^{\prime} \rightarrow \sigma} \int_{0}^{t}\left|\sigma_{r}^{\xi}-\sigma_{r}^{\prime \xi}\right|=0
$$


which completes the proof of Lemma 5.2.

Now we are ready to study the Green function for $L_{\gamma}$ in greater detail. Let

$$
G_{\gamma}(x, a ; y, b)=\int_{0}^{\infty} p_{t}(x, a ; y, b) d t
$$

The previous lemma, applied both to $L_{\gamma}$ and $L_{\gamma}^{*}$, says that $p_{t}(x, a ; y, b)$ is well defined also for $a \geq 0, b>0$ or for $a>0, b \geq 0$. Therefore $G_{\gamma}(x, a ; y, b)$ is defined for arbitrary $x, y$ in $N$ and $a^{2}+b^{2}>0$.

Theorem 5.6. $G_{\gamma}$ is the Green function for $L_{\gamma}$. More precisely,

$$
\begin{gathered}
G_{\gamma}(\cdot, \cdot ; y, b) \in L_{\mathrm{loc}}^{1}\left(N \times \mathbb{R}^{+}\right), \\
L_{\gamma} G_{\gamma}(\cdot, \cdot ; y, b)=-\delta_{(y, b)}, \\
G_{\gamma}(\cdot, \cdot ; y, b) \text { is a } L_{\gamma} \text {-potential, }
\end{gathered}
$$

and

$$
\begin{gathered}
G_{\gamma}(x, a ; \cdot, \cdot) \in L_{\mathrm{loc}}^{1}\left(N \times \mathbb{R}^{+}\right), \\
L_{\gamma}^{*} G_{\gamma}(x, a ; \cdot, \cdot)=-\delta_{(x, a)}, \\
G_{\gamma}(x, a ; \cdot, \cdot) \text { is a } L_{\gamma}^{*} \text {-potential. }
\end{gathered}
$$

In particular,

$$
\begin{aligned}
& L_{\gamma}^{*} G_{\gamma}(x, 0 ; \cdot, \cdot)=0 \text { on } N \times \mathbb{R}^{+}, \\
& L_{\gamma} G_{\gamma}(\cdot, \cdot ; y, 0)=0 \text { on } N \times \mathbb{R}^{+} .
\end{aligned}
$$

Finally, given $\varepsilon>0$, there exists $C>0$ such that

$$
C^{-1} \leq G_{\gamma}(x, a ; y, b) \leq C
$$

whenever $|x|<\varepsilon, 0 \leq a<\varepsilon,|y|=1, b \leq 1$ or $|y|<\varepsilon, 0 \leq b<\varepsilon$, $|x|=1, a \leq 1$, respectively. 
Proof. Since the heat semigroup $p_{t}^{*}(x, a ; y, b)$ corresponding to $L_{\gamma}^{*}$ is given by $p_{t}^{*}(x, a ; y, b)=p_{t}(y, b ; x, a)$ it is enough to prove (5.10)-(5.12). First we notice that

$$
\int_{0}^{\infty} T_{t} \phi(x, a) d t<\infty, \quad \text { for } \phi \in C_{0}^{\infty}\left(N \times \mathbb{R}^{+}\right)
$$

Indeed, if $t<1$ then $\left|T_{t} \phi(x, a)\right| \leq\|\phi\|_{L^{\infty}}$ and the beginning of the proof of Lemma 5.1 shows that

$$
\int_{1}^{\infty} T_{t} \phi(x, a) d t<\infty
$$

To prove (5.11) we write

$$
\begin{aligned}
& \int_{\mathbb{R}^{+}} \int_{N} L_{\gamma}^{*} G_{\gamma}(x, a ; y, b) \phi(y, b) d y b^{2 \alpha+1} d b \\
& \quad=\int_{\mathbb{R}^{+}} \int_{\mathbb{R}^{+}} \int_{N} p_{t}(x, a ; y, b) L_{\gamma} \phi(y, b) d y b^{2 \alpha+1} d b d t \\
& =\lim _{\substack{t_{1} \rightarrow 0 \\
t_{2} \rightarrow \infty}} \int_{t_{1}}^{t_{2}} \int_{\mathbb{R}^{+}} \int_{N} p_{t}(x, a ; y, b) L_{\gamma} \phi(y, b) d y b^{2 \alpha+1} d b d t
\end{aligned}
$$

because (5.16) is absolutely convergent. But

$$
\int_{\mathbb{R}^{+}} \int_{N} p_{t}(x, a ; y, b) L_{\gamma} \phi(y, b) d y b^{2 \alpha+1} d b=\partial_{t} T_{t} \phi(x, a) .
$$

Moreover,

$$
\lim _{t_{1} \rightarrow 0} T_{t_{1}} \phi(x, a)=-\phi(x, a)
$$

and by (4.9), Corollary 2.7, Lemma 2.2

$$
\left|T_{t_{2}} \phi(x, a)\right| \leq C \mathbf{E}_{a}\left(\int_{0}^{t_{2}} b^{\xi}(s) d s\right)^{-D / 2}
$$

which tends to 0 , when $t_{2} \rightarrow \infty$. This proves (5.11) and (5.13). To show that $G_{\gamma}(x, a ; \cdot, \cdot)$ is $L_{\gamma}^{*}$-potential we consider an $L_{\gamma}^{*}$-harmonic function $h$ satisfying

$$
0 \leq h(y, b) \leq G_{\gamma}(x, a ; y, b)
$$


and apply $T_{r}^{*}$ to it. Then, on one hand side

$$
T_{r}^{*} h(z, c)=h(z, c),
$$

and on the other,

$$
T_{r}^{*} h(z, c) \leq \int_{0}^{\infty} p_{t+r}(x, a ; z, c) d t \longrightarrow 0, \quad \text { for }(z, c) \neq(x, a)
$$

Hence $h=0$. (5.15) is a direct consequence of the next Lemma.

Lemma 5.18. Given $\xi>0, \alpha \geq 0, D>0, a_{1}>0$, there is $C$ such that if $a \leq a_{1}, 0<b<1,0<\eta<1$, then

$$
\begin{aligned}
& \int_{0}^{\infty} \mathbf{E}_{a}\left(\int_{0}^{t} b_{\alpha}^{\xi}(s) d s\right)^{-D / 2} e^{-c / A(0, t)} \\
& \cdot \mu([b-\eta, b+\eta])^{-1} \mathbf{1}_{\left\{b_{\alpha}: b_{\alpha}(t) \in[b-\eta, b+\eta]\right\}} d t<C,
\end{aligned}
$$

where $A(0, t)$ is defined in Theorem 4.1 and $\mu(A)=\int_{A} r^{2 \alpha+1} d r$.

Proof. Assume first that $t \geq 1$. Then, by the Markov property, it is enough to estimate

$$
\begin{aligned}
\int_{1}^{\infty} & \mathbf{E}_{a}\left(\int_{0}^{t / 2} b_{\alpha}^{\xi}(s) d s\right)^{-D / 2} \\
& \cdot \mu([b-\eta, b+\eta])^{-1} \mathbf{E}_{b_{\alpha}(t / 2)} \mathbf{1}_{\left\{\sigma_{\alpha}: \sigma_{\alpha}(t / 2) \in[b-\eta, b+\eta]\right\}}\left(\sigma_{\alpha}\right) .
\end{aligned}
$$

But by (2.1) and Lemma 2.3

$$
\mathbf{E}_{b_{\alpha}(t / 2)} \mathbf{1}_{\left\{\sigma_{\alpha}: \sigma_{\alpha}(t / 2) \in[b-\eta, b+\eta]\right\}}\left(\sigma_{\alpha}\right) \leq C t^{-1-\alpha} \mu([b-\eta, b+\eta]) .
$$

On the other hand by Lemma 2.2

$$
\begin{aligned}
& \mathbf{E}_{a}\left(\int_{0}^{t / 2} b_{\alpha}^{\xi}(s) d s\right)^{-D / 2} \\
& \quad=2^{(1+\xi / 2) D / 2} t^{-(1+\xi / 2) D / 2} \mathbf{E}_{a / \sqrt{t}}\left(\int_{0}^{1} b_{\alpha}^{\xi}(s) d s\right)^{-D / 2} .
\end{aligned}
$$

Now, Corollary 2.7 implies that (5.19) is dominated by a constant for every $a, b, \eta$. 
Let $t<1$. First we notice that for every $M, c>0$ there is $C$ such that $e^{-c / x} \leq C x^{M}$ for every $x>0$. Therefore, it suffices to estimate $\int_{0}^{1} \mathbf{E}_{a}\left(\int_{0}^{t} b_{\alpha}^{\xi}(s) d s\right)^{-D / 2} A(0, t) \mu([b-\eta, b+\eta])^{-1} \mathbf{1}_{\left\{b_{\alpha}: b_{\alpha}(t) \in[b-\eta, b+\eta]\right\}}$,

where

$$
A(0, t)=\int_{0}^{t}\left(b_{\alpha}^{m_{3}}(s)+b_{\alpha}^{m_{4}}(s)\right) d s
$$

Since

$$
A(0, t)^{M} \leq C\left(\left(\int_{0}^{t} b_{\alpha}^{m_{3}}(s) d s\right)^{M}+\left(\int_{0}^{t} b_{\alpha}^{m_{4}}(s) d s\right)^{M}\right)
$$

we are left with

$$
\begin{aligned}
I=\int_{0}^{1} & \mathbf{E}_{a}\left(\int_{0}^{t} b_{\alpha}^{\xi}(s) d s\right)^{-D / 2}\left(\int_{0}^{t} b_{\alpha}^{m_{j}}(s) d s\right)^{M} \\
& \cdot \mu([b-\eta, b+\eta])^{-1} \mathbf{1}_{\left\{b_{\alpha}: b_{\alpha}(t) \in[b-\eta, b+\eta]\right\}}\left(b_{\alpha}\right), \quad \xi, m_{j}>0
\end{aligned}
$$

and so, in view of the Schwartz inequality, we are to estimate

$$
I_{1}=\int_{0}^{1} \mathbf{E}_{a}\left(\int_{0}^{t} b_{\alpha}^{\xi}(s) d s\right)^{-D} \mathbf{1}_{\left\{b_{\alpha}: b_{\alpha}(t) \in[b-\eta, b+\eta]\right\}}\left(b_{\alpha}\right),
$$

and

$$
I_{2}=\int_{0}^{1} \mathbf{E}_{a}\left(\int_{0}^{t} b_{\alpha}^{m_{j}}(s) d s\right)^{2 M} \mathbf{1}_{\left\{b_{\alpha}: b_{\alpha}(t) \in[b-\eta, b+\eta]\right\}}\left(b_{\alpha}\right)
$$

By Lemma 2.2 and Corollary (2.15),

$$
\begin{aligned}
I_{1}= & t^{-(1+\xi / 2) D} \mathbf{E}_{a / \sqrt{t}}\left(\int_{0}^{1} b_{\alpha}^{\xi}(s) d s\right)^{-D} \\
& \cdot \mathbf{1}_{\left\{b_{\alpha}: b_{\alpha}(1) \in[(b-\eta) / \sqrt{t},(b+\eta) / \sqrt{t}]\right\}}\left(b_{\alpha}\right) \\
\leq & t^{-(1+\xi / 2) D} \mathbf{E}_{a / \sqrt{t}}\left(\int_{0}^{1 / 2} b_{\alpha}^{\xi}(s) d s\right)^{-D} \\
& \cdot \mathbf{E}_{b_{\alpha}(1 / 2)} \mathbf{1}_{\left\{\sigma_{\alpha}: \sigma_{\alpha}(1 / 2) \in[(b-\eta) / \sqrt{t},(b+\eta) / \sqrt{t}]\right\}}\left(\sigma_{\alpha}\right) \\
\leq & C t^{(1+\xi / 2) D-1-\alpha} \mu([b-\eta, b+\eta]) .
\end{aligned}
$$


Let $\Omega_{-1}=\left\{b_{\alpha}: \sup _{s \in[0,1]} b_{\alpha}(s) \leq a_{1}\right\}$ and

$$
\Omega_{m}=\left\{b_{\alpha}: a_{1}+m<\sup _{s \in[0,1]} b_{\alpha}(s) \leq a_{1}+m+1\right\}, \quad m=0,1,2, \ldots
$$

Then

$$
I_{2}=\sum_{m=-1}^{\infty} \mathbf{E}_{a}\left(\int_{0}^{t} b_{\alpha}^{m_{j}}(s) d s\right)^{2 M} \mathbf{1}_{\Omega_{m}}\left(b_{\alpha}\right) \mathbf{1}_{\left\{b_{\alpha}: b_{\alpha}(t) \in[b-\eta, b+\eta]\right\}}\left(b_{\alpha}\right) .
$$

We treat the cases $m=-1,0,1$ and $m \geq 2$ separately. For $m=-1,0,1$ we have

$$
\begin{array}{r}
\mathbf{E}_{a}\left(\int_{0}^{t} b_{\alpha}^{m_{j}}(s) d s\right)^{2 M} \mathbf{1}_{\Omega_{-1} \cup \Omega_{0} \cup \Omega_{1}}\left(b_{\alpha}\right) \mathbf{1}_{\left\{b_{\alpha}: b_{\alpha}(t) \in[b-\eta, b+\eta]\right\}}\left(b_{\alpha}\right) \\
\leq C t^{2 M-1-\alpha} \mu([b-\eta, b+\eta]) .
\end{array}
$$

Let $0<\sigma_{1}<1 / 2, A=\left(\sum_{n=1}^{\infty} 2^{-n \sigma_{1}}\right)^{-1}$ Then

$$
\Omega_{m} \subset \bigcup_{n=1}^{\infty} \bigcup_{k=1}^{2^{n}-1} \Omega_{m, n, k}
$$

where

$$
\Omega_{m, n, k}=\left\{b_{\alpha}: b_{\alpha}\left(\frac{k t}{2^{n}}\right)-b_{\alpha}\left(\frac{(k-1) t}{2^{n}}\right)>\frac{m A}{2^{n \sigma_{1}}}\right\} .
$$

Indeed, since $b_{\alpha}(t) \leq 2$ and $\sup _{s \in[0, t]} b_{\alpha}(s)>2$, we can always find $n$ and $k<2^{n}$ such that $b_{\alpha} \in \Omega_{m, n, k}$. Therefore, by Lemma (2.6),

$$
\begin{gathered}
\mathbf{E}_{a}\left(\int_{0}^{t} b_{\alpha}^{m_{j}}(s) d s\right)^{2 M} \mathbf{1}_{\Omega_{m, n, k}}\left(b_{\alpha}\right) \mathbf{1}_{\left\{b_{\alpha}: b_{\alpha}(t) \in[b-\eta, b+\eta]\right\}}\left(b_{\alpha}\right) \\
\cdot t^{2 M}\left(a_{1}+m+1\right)^{2 M m_{j}} \mathbf{E}_{a} \mathbf{1}_{\Omega_{m, n, k}}\left(b_{\alpha}\right) \mathbf{E}_{b_{\alpha}\left(k t / 2^{n}\right)} \\
\cdot \mathbf{1}_{\left\{\sigma_{\alpha}: s_{\alpha}\left(t-k t / 2^{n}\right) \in[b-\eta, b+\eta]\right\}}\left(\sigma_{\alpha}\right) \\
\leq C t^{2 M-1-\alpha}\left(a_{1}+m+1\right)^{2 M m_{j}} 2^{n(1+\alpha)} \mu([b-\eta, b+\eta] \\
\cdot \mathbf{E}_{a} \mathbf{E}_{b_{\alpha}\left(((k-1) t) / 2^{n}\right)} \mathbf{1}_{\left\{\sigma_{\alpha}: \sigma_{\alpha}\left(t / 2^{n}\right)>m A / 2^{n \sigma_{1}}+\sigma_{\alpha}(0)\right\}}\left(\sigma_{\alpha}\right) \\
\leq C t^{2 M-1-\alpha}\left(a_{1}+m+1\right)^{2 M m_{j}} 2^{n(1+\alpha)} \mu([b-\eta, b+\eta]) \\
\cdot \exp \left(-\frac{c_{2} m^{2} A^{2} 2^{n\left(1-2 \sigma_{1}\right)}}{t}\right)
\end{gathered}
$$


Hence,

$$
I_{2} \leq C t^{M-\alpha-1} \mu([b-\eta, b+\eta])
$$

and finally,

$$
I \leq C \int_{0}^{1} t^{-(1+\xi / 2)(D / 2)+M-\alpha-1} d t<+\infty
$$

Now we pass to the lower estimate for the Green function. Let $|y|=1, \eta>0$ and let $\phi_{\eta}$ be a family of smooth functions with the properties: $\operatorname{supp} \phi_{\eta} \subset\left\{z \in N:\left|y^{-1} z\right|<\eta\right\}, \phi_{\eta} \geq 0, \int \phi_{\eta}(z) d z=1$. Finally, let $\psi_{\eta}(\cdot)=\mu([b-\eta, b+\eta])^{-1} \mathbf{1}_{[b-\eta, b+\eta]}(\cdot)$.

Lemma 5.21. Given $a_{1}>0$ and a compact set $K \subset N$, there is $c>0$ such that for every $a \leq a_{1}, 0<b<1,0<\eta<1$,

$$
\int_{1}^{2} \mathbf{E}_{a} U^{b}(0, t) \varphi_{\eta}(x) \psi_{\eta}\left(b_{\alpha}(t)\right) d t \geq c, \quad x \in K
$$

Proof. Let $d, D$ be positive numbers which will be chosen later. We consider the set

$$
\Omega=\left\{b_{\alpha}: \sup _{s \in[0, t]} b_{\alpha}(s) \leq D, \inf _{s \in[t / 4,3 t / 4]} b_{\alpha}(s) \geq d\right\}
$$

and we estimate

$$
\int_{1}^{2} \mathbf{E}_{a} \varphi_{\eta} * p^{b}(t, 0)(x) \mathbf{1}_{\Omega}\left(b_{\alpha}\right) \mu([b-\eta, b+\eta])^{-1} \mathbf{1}_{\left\{b_{\alpha}: b_{\alpha}(t) \in[b-\eta, b+\eta]\right\}}\left(b_{\alpha}\right)
$$

from below. We have

$$
\begin{aligned}
\varphi_{\eta} & * p^{b}(t, 0)(x) \\
& =\iint \varphi_{\eta} * p^{b}\left(t, \frac{2 t}{3}\right)(z) p^{b}\left(\frac{2 t}{3}, \frac{t}{3}\right)\left(z^{-1} x y^{-1}\right) p^{b}\left(\frac{t}{3}, 0\right)(y) d z d y .
\end{aligned}
$$

In view of (4.7), we choose a compact set $K_{1}$ such that for $b \in \Omega$ and $1 \leq t \leq 2$,

$$
\int_{K_{1}} \varphi_{\eta} * p^{b}\left(t, \frac{2 t}{3}\right)(z) d z \geq \varepsilon>0, \quad \int_{K_{1}} p^{b}\left(\frac{t}{3}, 0\right)(y) d y \geq \varepsilon>0,
$$


where $\varepsilon=\varepsilon(A)$. Then, by Theorem (4.11) there is $C=C\left(D, d, K, K_{1}\right)$ such that

$$
p^{b}\left(\frac{2 t}{3}, \frac{t}{3}\right)\left(z^{-1} x y^{-1}\right) \geq C,
$$

for $z, y \in K_{1}, x \in K, b_{\alpha} \in \Omega, 1 \leq t \leq 2$. Therefore we are left with

$$
\begin{aligned}
I= & \mu([b-\eta, b+\eta])^{-1} \mathbf{P}_{a}\left(b_{\alpha}: b_{\alpha} \in \Omega, b_{\alpha}(t) \in[b-\eta, b+\eta]\right) \\
\geq & \mathbf{E}_{a} \mathbf{1}_{\left\{\sup _{s \in[0,2 t / 3]} b_{\alpha}(s) \leq D_{2}, \inf _{s \in[t / 3,2 t / 3]} b_{\alpha}(s) \geq d\right\}}\left(b_{\alpha}\right) \mu([b-\eta, b+\eta])^{-1} \\
& \cdot \mathbf{P}_{b_{\alpha}(2 t / 3)}\left(\sup _{s \in[0, t / 3]} \sigma_{\alpha}(s) \leq D, \sigma_{\alpha}\left(\frac{t}{3}\right) \in[b-\eta, b+\eta]\right)
\end{aligned}
$$

provided $D_{2}<D$. Notice that if $d \leq b_{\alpha}(2 t / 3) \leq D_{2}$,

$$
\mu([b-\eta, b+\eta])^{-1} \mathbf{P}_{b_{\alpha}(2 t / 3)}\left(\sigma_{\alpha}\left(\frac{t}{3}\right) \in[b-\eta, b+\eta]\right) \geq C=C\left(d, D_{2}\right) .
$$

But, proceeding as in the proof of the previous theorem we see that

$$
\begin{array}{r}
\mu([b-\eta, b+\eta])^{-1} \mathbf{P}_{b_{\alpha}(2 t / 3)}\left(\sup _{s \in[0, t / 3]} \sigma_{\alpha}(s) \geq D, \sigma\left(\frac{t}{3}\right) \in[b-\eta, b+\eta]\right) \\
\leq c_{1} e^{-c_{2}\left(D-D_{2}\right)^{2}} .
\end{array}
$$

Therefore choosing $D$ and $D_{2}$ appropriately we have

$$
\begin{array}{r}
\mu([b-\eta, b+\eta])^{-1} \mathbf{P}_{b_{\alpha}(2 t / 3)}\left(\sup _{s \in[0, t / 3]} \sigma_{\alpha}(s) \leq D, \sigma_{\alpha}\left(\frac{t}{3}\right) \in[b-\eta, b+\eta]\right) \\
\geq C\left(d, D, D_{2}\right),
\end{array}
$$

for $1 \leq t \leq 2$. Hence for $D_{1}<D_{2}$,

$$
\begin{aligned}
I \geq & C\left(d, D, D_{2}\right) \mathbf{E}_{a} \mathbf{1}_{\left\{b_{\alpha}: \sup _{s \in[0, t / 3]} b_{\alpha}(s) \leq D_{1}, b_{\alpha}(t / 3)>2 d\right\}} \\
& \cdot \mathbf{P}_{b_{\alpha}(t / 3)}\left(\inf _{s \in[0, t / 3]} \sigma_{\alpha}(s) \geq d, \sup _{s \in[0, t / 3]} \sigma_{\alpha}(s) \leq D_{2}\right) .
\end{aligned}
$$

By Lemmas 2.12 and 2.13

$$
\begin{aligned}
& \mathbf{P}_{b_{\alpha}(t / 3)}\left(\inf _{s \in[0, t / 3]} \sigma_{\alpha}(s) \geq d, \sup _{s \in[0, t / 3]} \leq D_{2}\right) \\
& \geq 1-\mathbf{P}_{b_{\alpha}(t / 3)}\left(\inf _{s \in[0, t / 3]} \sigma_{\alpha}(s)<d\right)-\mathbf{P}_{b_{\alpha}(t / 3)}\left(\sup _{s \in[0, t / 3]} \sigma_{\alpha}(s)>D_{2}\right) \\
& \geq 1-c_{1} e^{-c_{2} d^{2}}-c_{1} e^{-c_{2}\left(D_{2}-D_{1}\right)^{2}} \\
& \geq C>0
\end{aligned}
$$


provided $d$ and $D_{2}-D_{1}$ are large enough. Finally,

$$
\begin{aligned}
\mathbf{P}_{a}\left(\sup _{s \in[0, t / 3]} b_{\alpha}(s)\right. & \left.\leq D_{1}, b_{\alpha}\left(\frac{t}{3}\right)>2 d\right) \\
& \geq 1-\mathbf{P}_{a}\left(\sup _{s \in[0, t / 2]} b_{\alpha}(s)>D_{1}\right)-\mathbf{P}_{a}\left(b_{\alpha}\left(\frac{t}{3}\right)<2 d\right) \\
& \geq c_{1} e^{-c_{2} d^{2}}-c_{1} e^{-c_{2} D_{1}^{2}} \geq C>0,
\end{aligned}
$$

for sufficiently large $D_{1}$.

\section{Estimates of the Poisson kernels and the Martin boundary.}

(5.15) and (1.13) imply immediately the following estimates for $m_{\gamma}$.

Theorem 6.1. Let $m_{\gamma}$ be the Poisson kernel of $\mathcal{L}_{\gamma}, \gamma>0$. Then there exists a constant $C_{\gamma}$ such that

$$
C_{\gamma}^{-1}(|x|+1)^{-Q-\gamma} \leq m_{\gamma}(x) \leq C_{\gamma}(|x|+1)^{-Q-\gamma},
$$

for $x \in N$. In particular,

$$
C^{-1}(|x|+1)^{-Q} \leq m_{0}(x) \leq C(|x|+1)^{-Q},
$$

for $x \in N$.

Proof. Theorem 5.6 says that there is a positive constant $C_{\gamma}$ such that

$$
C_{\gamma}^{-1} \leq G_{-\gamma}(x, a ; e, 0) \leq C_{\gamma}
$$

if $|x|=1, a \leq 1$. Let $x=\sigma_{a}(y),|x|=a \geq 1,|y|=1$. By (1.18), we have

$$
\begin{aligned}
m_{\gamma}(x) & =G_{-\gamma}\left(x^{-1}, 1 ; e, 0\right) \\
& =G_{-\gamma}\left(\sigma_{a}(y), 1 ; e, 0\right) \\
& =a^{-Q-\gamma} G_{-\gamma}\left(y, a^{-1} ; e, 0\right) \\
& =|x|^{-Q-\gamma} G_{-\gamma}\left(y, a^{1} ; e, 0\right),
\end{aligned}
$$


and the proof is completed.

Now we consider the case $\gamma=0$, i.e. we look at the operator $\mathcal{L}_{0}$. The next theorem gives description of the Martin boundary for $\mathcal{L}_{0}$.

Theorem 6.3. The Martin boundary for $\mathcal{L}=\mathcal{L}_{0}$ consists of the following functions:

a) the constant function 1 ,

b) $P_{y}(x a)=\frac{1}{m_{0}(e)} a^{-Q} \breve{m}_{0}\left(\sigma_{a^{-1}}\left(y^{-1} x\right)\right)$.

All of them are minimal.

Proof. By (1.17) we may use $G$ to write the Martin kernels. Assume that

$$
\lim _{n \rightarrow \infty} \frac{G\left(x, a ; y_{n}, b_{n}\right)}{G\left(e, 1 ; y_{n}, b_{n}\right)}=K(x, a)
$$

and $\left|y_{n}\right| \longrightarrow \infty$ or $b_{n} \longrightarrow \infty$.

Let $r_{n}=\max \left\{\left|y_{n}\right|, b_{n}\right\}$. Then

$$
G\left(x, a ; y_{n}, b_{n}\right)=r_{n}^{-Q} G\left(\sigma_{r_{n}^{-1}}(x), r_{n}^{-1} a ; \sigma_{r_{n}^{-1}}\left(y_{n}\right), r_{n}^{-1} b_{n}\right) .
$$

We take $n$ such that

$$
\left|\sigma_{r_{n}^{-1}}(x)\right|<\frac{1}{4}, \quad r_{n}^{-1} a<\frac{1}{4} .
$$

Since $\left|\sigma_{r_{n}^{-1}}\left(y_{n}\right)\right|=1$ and $r_{n}^{-1} b_{n} \leq 1$ or $\sigma_{r_{n}^{-1}}\left(y_{n}\right) \leq 1$ and $r_{n}^{-1} b_{n}=1$, by Theorem 5.4 and the Harnack inequality for $L^{*}$, there is a constant $c$ independent of $x, a$ such that

$$
\begin{gathered}
c^{-1} \leq G\left(\sigma_{r_{n}^{-1}}(x), r_{n}^{-1} a ; \sigma_{r_{n}^{-1}}\left(y_{n}\right), r_{n}^{-1} b_{n}\right) \leq c, \\
c^{-1} \leq G\left(e, r_{n}^{-1} ; \sigma_{r_{n}^{-1}}\left(y_{n}\right), r_{n}^{-1} b_{n}\right) \leq c .
\end{gathered}
$$

Therefore $K(x, a)$ is bounded and so must be constant (see [BR]).

Now we assume that $y_{n} \longrightarrow y_{0}$ and $b_{n} \longrightarrow 0$. First we prove that

$$
\lim _{n \rightarrow \infty} \frac{G\left(x, a ; y_{n}, b_{n}\right)}{G\left(e, 1 ; y_{n}, b_{n}\right)}=\lim _{n \rightarrow \infty} \frac{G\left(y_{0}^{-1} x, a ; e, b_{n}\right)}{G\left(e, 1 ; e, b_{n}\right)}
$$


i.e. that

$$
\lim _{n \rightarrow \infty} \frac{G\left(y_{n}^{-1} x, a ; e, b_{n}\right)}{G\left(y_{0}^{-1} x, a ; e, b_{n}\right)}=1
$$

Notice that for $n$ sufficiently large (depending on $x, a), \tau\left(y_{n}^{-1} x, a\right.$; $\left.y_{0}^{-1} x, a\right)<1$. Hence by the Harnack inequality

$$
\begin{aligned}
\left|G\left(y_{n}^{-1} x, a ; e, b_{n}\right)-G\left(y_{0}^{-1} x, a ; e, b_{n}\right)\right| & \\
& \leq G\left(y_{0}^{-1} x, a ; e, b_{n}\right) \tau\left(y_{n}^{-1} x, a ; y_{0}^{-1} x, a\right) .
\end{aligned}
$$

and (6.5) follows. We have

$$
G\left(x, a ; e, b_{n}\right)=a^{-Q} G\left(\sigma_{a^{-1}}(x), 1 ; e, a^{-1} b_{n}\right) .
$$

Therefore when $b_{n} \longrightarrow 0$,

$$
\lim _{b_{n} \rightarrow 0} G\left(x, a ; e, b_{n}\right)=a^{-Q} G\left(\sigma_{a^{-1}}(x), 1 ; e, 0\right)=a^{-Q} \breve{m}\left(\sigma_{a^{-1}}(x)\right)
$$

and so

$$
\lim _{b_{n} \rightarrow 0} \frac{G\left(x, a ; e, b_{n}\right)}{G\left(e, 1 ; e, b_{n}\right)}=\frac{1}{m_{0}(e)} a^{-Q} \breve{m}_{0}\left(\sigma_{a^{-1}}(x)\right)=P_{e}(x a) .
$$

1 is minimal because the only bounded $\mathcal{L}$-harmonic functions are constants, $P_{e}$ is minimal if and only if $P_{y}$ is minimal. Hence all of them are minimal.

Acknowledgements. The authors are very grateful to Jacek Zienkiewicz whose ideas are incorporated in one form or another in the paper. The authors would like to express their gratitude to Didier Arnal, Bashir Bekka and Jean Ludwig for their hospitality and their interest in the subject of this paper at its various stages.

\section{References.}

[A] Ancona, A., Negatively curved manifolds, elliptic operators, and the Martin boundary. Ann. Math. 125 (1987), 495-535. 
[AS] Anderson, M., Schoen, R., Positive harmonic functions on complete manifolds of negative curvature. Ann. of Math. 121 (1985), 429-461.

[B] Babillot, M., Comportement asymptotique du mouvement brownien sur une variété homogène à courboure négative ou nulle. Ann. Inst. $H$. Poincaré 27 (1991), 61-90.

[BR] Birgé, L., Raugi, A., Fonctions harmoniques sur les groupes moyennables. C. R. Acad. Sci. Paris 278 (1974), 1287-1289.

[D1] Damek, E., Left-invariant degenerate elliptic operators on semidirect extensions of homogeneous groups. Studia Math. 89 (1988), 169-196.

[D2] Damek, E., Pointwise estimates for the Poisson kernel on $N A$ groups by the Ancona method. Ann. de Toulouse V (1996), 421-441.

[DH1] Damek, E., Hulanicki, A., Maximal functions related to subelliptic operators invariant under an action of a nilpotent group. Studia Math. 103 (1992), 239-264.

[DH2] Damek, E., Hulanicki, A., Boundaries for left-invariant subelliptic operators on semidirect products of nilpotent and abelian groups. J. reine angew. Math. 411 (1990), 1-38.

[DHZ] Damek, E., Hulanicki, A., Zienkiewicz, J., Estimates for the Poisson kernels and their derivatives on rank one $N A$ groups. Studia Math. 126 (1997), 114-148.

[E] Élie, L., Comportement asymptotique du noyau potentiel sur les groupes de Lie. Ann. Sci. École Norm. Sup. 15 (1982), 257-364.

[G] Guivarc'h, Y., Quelques properiétés asymptotiques des produits de matrices aléatoires. Lecture Notes in Math. 774 (1980), 177-250. SpringerVerlag.

[H] Hulanicki, A., Subalgebra of $L_{1}(G)$ associated with Laplacian on a Lie group. Colloq. Math. 31 (1974), 259-287.

[Hei] Heintze, E., On homogeneous manifolds of negative curvature. Math. Ann. 211 (1974), 23-34.

[K] Kiefer, Y., Brownian motion and positive harmonic functions on complete manifolds of non-positive curvature. From local times to global geometry, control and physics. Longman Scientific \& Technical (1984/85), $187-232$.

[L] Lebedev, N. N., Special functions and their applications. Dover Publications, Inc., 1972.

[M] Malliavin, P., Géométrie Différentielle Stochastique. Seminaire de Mathématique Supérieur Été 1997 No 64. Les Press de l'Université de Montréal, 1978. 
[MM] Malliavin, M. P., Malliavin, P., Factorisation et lois limites de la diffusion horizontale au-dessus d'un espace riemannien symétrique. Lecture Notes in Math. 404 (1974), 164-217. Springer-Verlag.

[Mo] Molchanov, S. A., Martin boundary for invariant Markov processes on a solvable group. Theor. Probab. Appl. 12 (1967), 310-314.

[Ra] Raugi, A., Fonctions harmoniques sur les groupes localement compacts à base dénombrable. Bull. Soc. Math. France, Mémoire. 54 (1977), $5-118$.

[RY] Revuz, D., Yor, M., Continuous Martingales and Brownian Motion. Springer-Verlag, 1991.

[S] Stroock, D. W., personal communication.

[SS] Saloff-Coste, S., Stroock, D. W., Opérateurs uniformément sous-elliptiques sur les groupes de Lie. J. Funct. Anal. 98 (1991), 97-121. Academic Press.

[T] Tanabe, H., Equations of Evolution. Pitman, 1979.

[Tay] Taylor, J. C., Skew products, regular conditional probabilities and stochastic differential equations: a technical remark. In Séminaire de Probabilité XXVI, Lecture Notes in Math. 1526 (1992), 299-314. SpringerVerlag.

[VSC] Varopoulos, N. Th., Saloff-Coste, L., Coulhon, T., Analysis and Geometry on Groups. Cambridge Tracts in Math. 100, Cambridge Univ. Press, 1992.

[W] Wolf, J. A., Homogeneity and bounded isometries in manifolds of negative curvature. Illinois J. Math. 8 (1964), 14-18.

Recibido: 26 de julio de 1.999

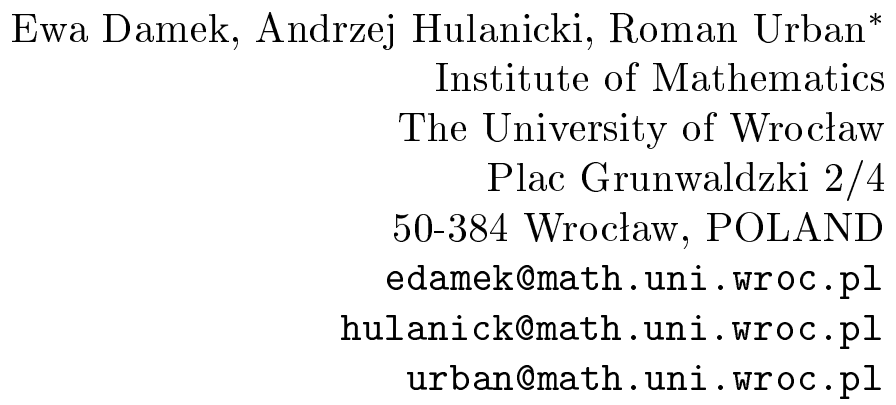

* The authors were partly supported by KBN grant 2P03A04316, by the European Commission via the TMR network "Harmonic Analysis", contract no. ERB FMRXCT97-0159 and Foundation for Polish Sciences, Subsidy 3/99 